\title{
Oleanolic Acid Inhibits Neuronal Pyroptosis in Ischaemic Stroke by Inhibiting miR-186-5p Expression
}

\author{
Shi-Chang $\mathrm{Cai}^{1 \dagger}$, Xiu-Ping $\mathrm{Li}^{2 \dagger}$, Xing $\mathrm{Li}^{3 \dagger}$, Gen-Yun Tang ${ }^{4}$, Li-Ming $\mathrm{Yi}^{1}$ and Xiang-Shang $\mathrm{Hu}^{1 *}$ \\ ${ }^{1}$ Department of Human Anatomy, School of Basic Medical Sciences, Hunan University of Medicine, Huaihua 418000, ${ }^{2}$ School \\ of Public Health and Laboratory Medicine, Hunan University of Medicine, Huaihua 418000, ${ }^{3}$ School of Basic Medical \\ Sciences, Shaoyang University, Shaoyang 422000, ${ }^{4}$ Department of Cell Biology and Genetics, School of Basic Medical Sciences, \\ Hunan University of Medicine, Huaihua 418000, Hunan Province, P.R. China
}

\begin{abstract}
Ischaemic stroke is a common condition leading to human disability and death. Previous studies have shown that oleanolic acid (OA) ameliorates oxidative injury and cerebral ischaemic damage, and miR-186-5p is verified to be elevated in serum from ischaemic stroke patients. Herein, we investigated whether OA regulates miR-186-5p expression to control neuroglobin ( $\mathrm{Ngb}$ ) levels, thereby inhibiting neuronal pyroptosis in ischaemic stroke. Three concentrations of $\mathrm{OA}(0.5,2$, or $8 \mu \mathrm{M})$ were added to primary hippocampal neurons subjected to oxygen-glucose deprivation/ reperfusion (OGD/R), a cell model of ischaemic stroke. We found that OA treatment markedly inhibited pyroptosis. qRT-PCR and western blot revealed that OA suppressed the expression of pyroptosis-associated genes. Furthermore, OA inhibited LDH and proinflammatory cytokine release. In addition, miR-186-5p was downregulated while Ngb was upregulated in OA-treated OGD/R neurons. MiR-186-5p knockdown repressed OGD/R-induced pyroptosis and suppressed LDH and inflammatory cytokine release. In addition, a dual luciferase reporter assay confirmed that miR-186-5p directly targeted Ngb. OA reduced miR-186-5p to regulate Ngb levels, thereby inhibiting pyroptosis in both OGD/R-treated neurons and MCAO mice. In conclusion, OA alleviates pyroptosis in vivo and in vitro by downregulating miR-186-5p and upregulating Ngb expression, which provides a novel theoretical basis illustrating that $\mathrm{OA}$ can be considered a drug for ischaemic stroke.
\end{abstract}

Key words: Ischaemic stroke, Oleanolic acid, miR-186-5p, Neuroglobin, Pyroptosis

\section{INTRODUCTION}

Ischaemic stroke is one of the major causes of long-term disability and death worldwide, leading to sudden onset of focal neurological function loss due to infarction in the relevant part of the brain [1]. Ischaemic stroke is induced by an abrupt and sustained decrease in regional cerebral blood flow resulting in cerebral hypoxia/ischaemia [2]. Early events after ischaemic damage generate glutamate-induced excitotoxicity and reactive oxygen species

Submitted March 22, 2021, Revised November 26, 2021,

Accepted December 2, 2021

* To whom correspondence should be addressed.

TEL: 86-13974582851, FAX: 86-13974582851

e-mail:yzhxs@126.com

These authors contributed equally to this article.
(ROS)-mediated oxidative stress, both of which accelerate cell death within the infarct core, an inflammatory cascade of events escalating progressive damage [3]. Clinical trials have shown that endovascular thrombectomy, intravenous thrombolytic therapy and several drugs, such as neurotransmission modulators, antiinflammatory agents and free radical scavengers, are crucial cytoprotective components for ischaemic stroke treatment $[4,5]$. However, in stroke, the main problem is neuronal cell loss, making functional recovery difficult; therefore, it is of great significance to explore possible novel molecular and cellular treatment options to prevent neuronal loss in ischaemic stroke.

Emerging evidence suggests that oleanolic acid (OA), a pentacyclic triterpenoid extracted from various medicinal plants, plays significant roles in health by affecting anti-inflammatory and antioxidant activities $[6,7]$. OA has been recognized as a neuro-
Copyright (c) Experimental Neurobiology 2021. www.enjournal.org
This is an Open Access article distributed under the terms of the Creative Commons Attribution Non-Commercial License (http://creativecommons.org/licenses/by-nc/4.0) which permits unrestricted non-commercial use, distribution, and reproduction in any medium, provided the original work is properly cited. 
protective strategy in numerous neurodegenerative disorders [8]. A previous study demonstrated that OA suppresses apoptosis and the inflammatory response to protect against spinal cord injury [9]. In addition, OA ameliorates carotid artery injury in diabetic rats via reduction of the Nod-like receptor protein 3 (NLRP3) inflammasome signalling pathway [10]. Furthermore, OA remarkably decreases oxidative injury in vitro and cerebral ischaemic damage in vivo [11]. However, the accurate neuroprotective role and potential mechanism of OA in ischaemic stroke have yet to be elucidated.

Increasing evidence has illustrated that neuroglobin (Ngb), an oxygen-binding globin protein that is specifically expressed in neurons, plays an endogenous neuroprotective role against oxidative stress-related and hypoxic/ischaemic injury in neurodegenerative disorders and stroke [12]. Studies have revealed that $\mathrm{Ngb}$ is upregulated in ischaemic neurons of mouse and human brains, and Ngb knockdown suppresses axonal regeneration in OGD/R-treated neurons [13]. Interestingly, overexpression of Ngb promotes neurogenesis in mouse brains following MCAO [14]. Moreover, Ngb mediates the neuroprotective effect of hypoxic postconditioning (HPC) against rat cerebral ischaemia [15]. Pyroptosis is a form of programmed cell death characterized by DNA fragmentation, plasma membrane rupture, cell swelling and the release of proinflammatory factors [16]. Mechanistically, caspase-1 is activated by the NLRP3/ASC pathway and inflammasomes, which mediate the cleavage of gasdermin D (GSDMD), subsequently triggering pyroptosis $[17,18]$. Recent studies have revealed that pyroptosis is involved in the pathogenesis of ischaemic stroke and that suppression of pyroptosis alleviates ischaemic brain damage $[19,20]$. A recent study indicated that Mn-TAT PTD-Ngb, a new artificial recombinant protein, inhibits inflammasome assembly, suppresses proinflammatory cytokine production, including IL-1 $\beta$ and IL-18, safeguards against oxidative stress and elevates pyroptosis [21]. However, whether Ngb and pyroptosis are implicated in the effects of $\mathrm{OA}$ on ischaemic stroke remains unknown.

It has been reported that $\mathrm{OA}$ ameliorates disease severity through the regulation of microRNAs (miRNAs). For instance, OA induces increased miR-132, which acts as a vital modulator to ameliorate chronic unpredictable mild stress (CUMS)-induced anhedonic and anxiogenic behaviours [22]. Interestingly, in hyperlipidaemic mice induced by a high-fat diet, OA ameliorates hyperlipidaemia, possibly by regulating the miR-98-5p/peroxisome proliferator-activated receptor- $\gamma$ coactivator- $1 \beta$ (PGC-1 $\beta$ ) axis [23]. As reported by various studies in recent years, miRNAs play key roles in stroke pathogenesis as well as its complications and outcomes [24]. It has been verified that miR-186-5p is elevated in serum from ischaemic stroke patients compared to healthy donors and facilitates apopto- sis of SH-SY5Y cells by targeting insulin-like growth factor (IGF1) after $\mathrm{OGD} / \mathrm{R}$ [25]. The targeted binding relationship between miR-186-5p and Ngb was predicted by bioinformatics analysis using the TargetScan website (http://www.targetscan.org/vert_72/). Therefore, we speculated that OA regulates miR-186-5p to affect Ngb expression, thereby inhibiting neuronal pyroptosis to exert a neuroprotective effect in ischaemic stroke.

To test our hypothesis, we first investigated whether administration of OA could protect against ischaemia/reperfusion-induced neuronal damage in MCAO mice and OGD/R-induced neurons. We found that OA treatment alleviated OGD/R-induced pyroptosis, cytotoxicity, and proinflammatory cytokine release and suppressed the expression of pyroptosis-associated proteins. In addition, OA relieved pyroptosis and subsequently rescued the biological functions of neurons by increasing $\mathrm{Ngb}$ and reducing miR-186-5p expression. To the best of our knowledge, our study is the first to demonstrate the pyroptosis-inhibiting effect of OA in ischaemic stroke through a miRNA-dependent mechanism, which suggests that OA might have therapeutic potential for ischaemic stroke patients.

\section{MATERIALS AND METHODS}

\section{Cell culture}

Primary hippocampal neurons were harvested from C57BL/6 mouse embryos at 14 15 d as previously described [26]. Mice were euthanized with cervical dislocation under anaesthesia. After collection on a cold stage, primary hippocampal neurons were dissociated into a single-cell suspension in Dulbecco's modified Eagle medium (DMEM, Gibco, Grand Island, NY, USA) containing foetal bovine serum (FBS, Gibco). The suspension ( 1 or $2 \times 10^{5}$ cells/ $\mathrm{cm}^{2}$ ) was plated on polylysine-coated plates. Neurobasal medium (0.5 mM glutamine, 2\% B27, 1\% penicillin-streptomycin) was used to substitute the culture medium. Then, the cells were cultured in a humidified incubator at $37^{\circ} \mathrm{C}$ in a $5 \% \mathrm{CO}_{2}$ atmosphere. The hippocampal neurons required $7 \sim 8$ days for maturation, and the medium was replaced every 3 days. The culture was confirmed to contain $90 \%$ neurons using immunocytochemistry for antiNF200.

\section{Animals and construction of middle cerebral artery occlusion (MCAO)}

Adult male C57BL/6J mice ( $8 \sim 10$ weeks) were acquired from the Animal Centre of the Chinese Academy of Sciences (Shanghai, China). All mice were housed in a quiet environment with free accessed to food and water. The environment was kept at $22 \pm 2^{\circ} \mathrm{C}$ and stable humidity with a $12 \mathrm{~h}$ light/dark cycle. All experimental 
procedures were performed strictly in line with animal care using the introduction of the American Animal Protection Legislation and were approved by the Ethics Committee of Hunan University of Medicine.

A middle cerebral artery occlusion (MCAO) model in mice was constructed using intraluminal vascular occlusion and subsequent reperfusion. In brief, mice were subcutaneously anaesthetized with chloral hydrate $(350 \mathrm{mg} / \mathrm{kg}$, ip). The vessels of the neck were uncovered, and the right common carotid arteries, external carotid arteries and internal carotid arteries were carefully separated. A 5-0 nylon filament with a round tip ( $0.23 \mathrm{~mm}$ in diameter) [27-29] was inserted into the internal carotid arteries to block the origin of the middle cerebral artery for $1.5 \mathrm{~h}$. After the operation, ischaemic artery reperfusion was established with filament withdrawal, followed by reperfusion for $24 \mathrm{~h}$. Mice in the sham group underwent the same procedures except for occlusion of the internal carotid artery. The mice were randomly divided into three groups ( $\mathrm{n}=6$ in each group, three batches were performed simultaneously in each group: mice in one batch were used for TTC staining; another batch was used to detect the expression levels of protein and gene by western blot assay and qRT-PCR; the last batch was used to detect $\mathrm{LDH}$ and proinflammatory cytokines release): sham group (without MCAO), MCAO group and $\mathrm{MCAO}+\mathrm{OA}$ group. Mice in the MCAO+OA group were treated with DMSO-diluted OA (25 $\mathrm{mg} / \mathrm{kg}$, Chengdu Purechem-Standard, Chengdu, China) by intraperitoneal injection once daily for four consecutive days prior to surgery.

\section{Oxygen-glucose deprivation/reperfusion $(O G D / R)$ in vitro model}

The OGD/R model was constructed in hippocampal neurons as previously described [26]. For OGD/R, cells were treated in medium for $8 \mathrm{~h}$ at $37^{\circ} \mathrm{C}$, with deprivation of serum and glucose within an oxygen-free environment $\left(5 \% \mathrm{CO}_{2}\right.$ and $\left.95 \% \mathrm{~N}_{2}\right)$ at $37^{\circ} \mathrm{C}$. Afterwards, for reperfusion, cells received normal medium in a normoxic environment for $24 \mathrm{~h}$. In the OA treatment groups, OA was added to the cells at final concentrations of $0.5,2$ and $8 \mu \mathrm{M}$ for $1 \mathrm{~h}$ before OGD/R. In the control groups, the same volume of PBS without $\mathrm{OGD} / \mathrm{R}$ or $\mathrm{OA}$ was added to the cells.

\section{Cell transfection}

The miR-186-5p inhibitor, miR-186-5p mimics and negative controls (NCs) were synthesized by KeyGen BioTech (Nanjing, China). p-Ngb (Ngb overexpression vector) and pcDNA3.1 were generated from Sangon Biotech (Shanghai, China). All transfection procedures were conducted using Lipofectamine 2000 (Life Technologies, Carlsbad, CA, USA) according to the manufacturer's instructions for $48 \mathrm{~h}$.

\section{2,3,5-Triphenyltetrazolium chloride (TTC) staining}

Mice were sacrificed under anaesthesia after the MCAO procedure for $24 \mathrm{~h}$. Brain samples were transferred to precooled stainless steel brain matrices and frozen at $-20^{\circ} \mathrm{C}$ for $10 \mathrm{~min}$. Subsequently, tissues were sectioned into five $2 \mathrm{~mm}$ thick sections and immersed in 2.0\% TTC solution (Sigma-Aldrich, St. Louis, MO, USA) at $37^{\circ} \mathrm{C}$ for $0.5 \mathrm{~h}$. After washing with PBS three times, $10 \%$ formalin was used to fix these sections. Slices were visualized using digital camera, and infarct volume was measured.

\section{Quantitative real-time polymerase chain reaction (qRT-PCR)}

Total RNA from cells or tissues was harvested using TRIzol reagent (BBI, Shanghai, China). RNA solution ( $1 \mu \mathrm{l})$ was used for RNA quality and concentration tests on a NanoDrop (Thermo Fisher, Waltham, MA, USA). First-strand cDNA was synthesized using a Bestar qPCR RT kit (DBIBioscience, Germany) or TaqMan miRNA Reverse Transcription kit (Thermo Fisher) following the manufacturer's guidelines. Expression of miR-186-5p was determined using a TaqMan miRNA assay (Applied Biosystems, Foster, CA, USA), and U6 was adopted as an internal reference. For Ngb mRNA measurement, a SYBR Green PCR kit (Thermo Fisher) was utilized. In accordance with the amplification procedure, an ABI 7500 Fast Real-Time PCR system (Applied Biosystems, Life Technologies) was applied for all PCRs. $\beta$-actin was adopted as an internal reference, and the $2^{-\Delta \Delta \mathrm{Ct}}$ method was utilized to determine relative gene expression.

Primers sequences were as follows:

miR-186-5p, forward, 5'-AAGAATTCTCCTTTTGGGCT-3', and reverse, 5'-GTGCGTGTCGTGGAGTCG -3';

Ngb, forward, 5'-CTCTGGAACATGGCACTGTC-3', and reverse, 5'-TTGGTCACTGCAGCATCAAT-3';

U6, forward, 5'-GCACATATACGCTTCGGCATAAAAT-3', and reverse, 5'-CATTTGCGGCTTCACGATGTCAT-3';

$\beta$-actin, forward, 5'-TGTCACCAACTGGGACGATA-3', and reverse, 5'-GGGGTGTTGAAGGTCTCAAA-3'.

\section{Western blot analysis}

Proteins were separated from cells or tissues using RIPA buffer (EMD Millipore, Billerica, MA, USA). Then, protein concentrations were determined using a bicinchoninic acid (BCA) protein assay kit (Thermo Fisher). The same concentration of samples was separated by $10 \%$ sodium dodecyl sulphate-polyacrylamide gel electrophoresis (SDS-PAGE) and then transferred to polyvinylidene fluoride (PVDF) membranes (Bio-Rad Laboratories Inc., 
Hercules, CA, USA). Next, membranes were blocked with 5\% nonfat dry milk at room temperature for $2 \mathrm{~h}$. Then, they were incubated at $4{ }^{\circ} \mathrm{C}$ for $24 \mathrm{~h}$ with the following primary antibodies: antiNLRP3 (1:500, Abcam, Cambridge, MA, USA), anti-pro-caspase-1 (1:1,000, Abcam), anti-cleaved-caspase-1 (1:500, Adipogen, San Diego, California, USA), anti-GSDMD-FL (1:500, Abcam), antiGSDMD-N (1:500, Abcam), anti-Ngb (1:500, Abcam), and antiGAPDH (1:1,000, Abcam). At room temperature, the membranes were then incubated with horseradish peroxidase (HRP)-conjugated secondary antibodies for $2 \mathrm{~h}$. An enhanced chemiluminescence (ECL) kit (Bio-Rad, USA) was used to image the protein bands.

\section{Flow cytometry}

For pyroptosis examination, flow cytometry was utilized with caspase- 1 and propidium iodide (PI) staining. Following washing twice with phosphate-buffered saline (PBS), cells were harvested and incubated for $0.5 \mathrm{~h}$ with caspase- 1 antibody (1:500, Adipogen) at room temperature. Subsequently, they were treated with secondary antibody for $0.5 \mathrm{~h}$ in the dark at room temperature. Next, samples were stained with PI from an Annexin V-FITC Apoptosis Detection kit (Pharmingen-Becton Dickinson, San Diego, CA, USA) in accordance with the manufacturer's guidelines. The experiment was performed using a flow cytometer (Attune NxT, Thermo Fisher) and was repeated at least three times.

\section{Enzyme-linked immunosorbent assay (ELISA)}

Brain tissues collected from mice were homogenized in $500 \mu \mathrm{l}$ of 0.1 M PBS. Next, the tissues were centrifuged at 10,000 rpm for 20 min at $4{ }^{\circ} \mathrm{C}$. In addition, cell supernatants were obtained from hippocampal neurons by centrifugation at $10,000 \mathrm{rpm}$ for $15 \mathrm{~min}$. IL$1 \beta$ and IL-18 (EK0393) in brain tissues and cell supernatants were analysed using ELISA kits (Kehua Biotechnology Ltd., Shanghai, China) following the manufacturer's instructions. In brief, samples and IL- $1 \beta$ or IL- 18 standards were added to the wells and incubated for $2 \mathrm{~h}$. After that, streptavidin-horseradish peroxidase (HRP) $(100 \mu \mathrm{l})$ was added for $20 \mathrm{~min}$. Plates were washed with PBS, and then substrate solution $(100 \mu \mathrm{l})$ was added and incubated for 20 min. Finally, stop solution $(50 \mu \mathrm{l})$ was added to each well, and the plate was gently mixed. The optical density of each well was immediately examined at $450 \mathrm{~nm}$ using a microplate reader (Bio-Rad, Hercules, CA, USA).

\section{LDH release assay}

Lactate dehydrogenase (LDH), a marker of membrane integrity, was detected to evaluate cytotoxicity using the LDH Activity Assay kit (Biovision, Tucson, AZ, USA). Briefly, the cultured cells were plated in 96-well plates, and brain tissues were made into brain homogenates after centrifugation at 3,000 rpm for $10 \mathrm{~min}$, followed by the addition of $\mathrm{LDH}$ reaction solution $(100 \mu \mathrm{l} /$ well $)$ for $30 \mathrm{~min}$. Subsequently, the absorbance was monitored by a microplate reader (Bio-Tek Instruments, Inc., Winooski, VT, USA) at $490 \mathrm{~nm}$.

\section{Luciferase reporter assay}

The predicted binding site of miR-186-5p and Ngb was obtained from TargetScan version 7.2 (http://www.targetscan.org/vert_72/). The 3'-UTR (untranslated region) of Ngb mRNA containing miR-186-5p binding sites was amplified and inserted into the pmirGLO vector (Promega, Madison, WI, USA) to construct wildtype pmirGLO-Ngb (WT-Ngb). Meanwhile, the pmirGLO-Ngb 3'-UTR-mutant (MUT-Ngb) with mutation of predicted miR186-5p binding sites was constructed using a QuikChange Site-directed Mutagenesis kit (Stratagene, La Jolla, CA, USA). Afterwards, hippocampal neurons were cotransfected with luciferase reporter plasmid (200 ng) and $50 \mathrm{nM}$ of the miR-186-5p mimics or mimics NC with Lipofectamine 2000 (Life Technologies, Carlsbad, CA, USA). Luciferase activity was assessed using the dual luciferase reporter assay system (Promega). Renilla luciferase was utilized to calculate the relative luciferase activity.

\section{Statistical analysis}

All data were analysed using SPSS 20.0 software and are presented as the mean \pm standard deviation (SD). All experiments were completed in triplicate. Quantitative data were determined by Student's t-test for two groups, while multiple comparisons were analysed using one-way ANOVA with Tukey's post hoc test. Results were considered statistically significant at $\mathrm{p}<0.05$.

\section{Ethical Approval}

All experimental procedures were in line with animal care strictly and used introduction of the American Animal Protection Legislation and were approved by the Ethics Committee of Hunan University of Medicine.

\section{RESULTS}

\section{OGD/R treatment leads to hippocampal neuron pyroptosis}

To investigate the impact of OGD/R on primary hippocampal neurons, pyroptosis was evaluated by flow cytometry using PI and caspase-1 staining. The results showed that pyroptosis was aggravated in hippocampal neurons subjected to OGD/R (Fig. 1A). Furthermore, ELISA results showed that the proinflammatory cytokines IL-1 $\beta$ and IL-18 were increased in the OGD/R group (Fig. 1B). Additionally, LDH release in the supernatant was used to esti- 
A

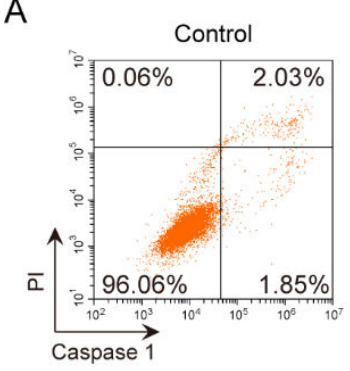

C

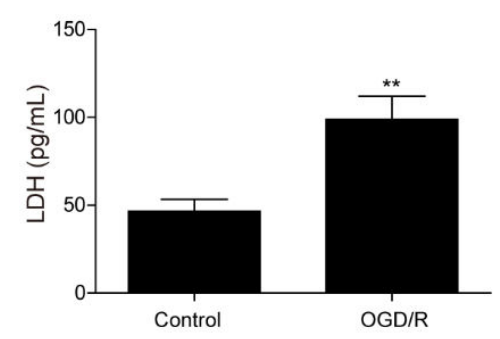

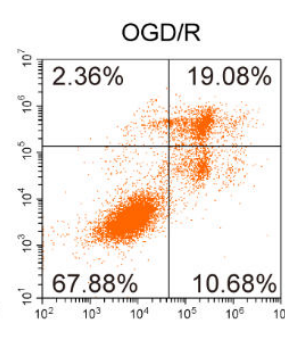

$\mathrm{D}$

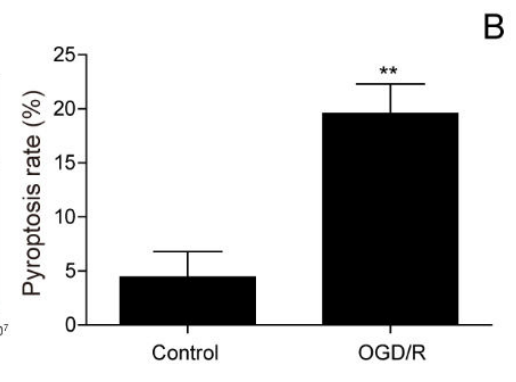

Control
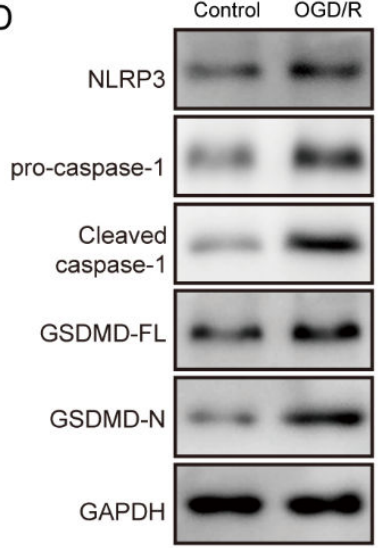

B
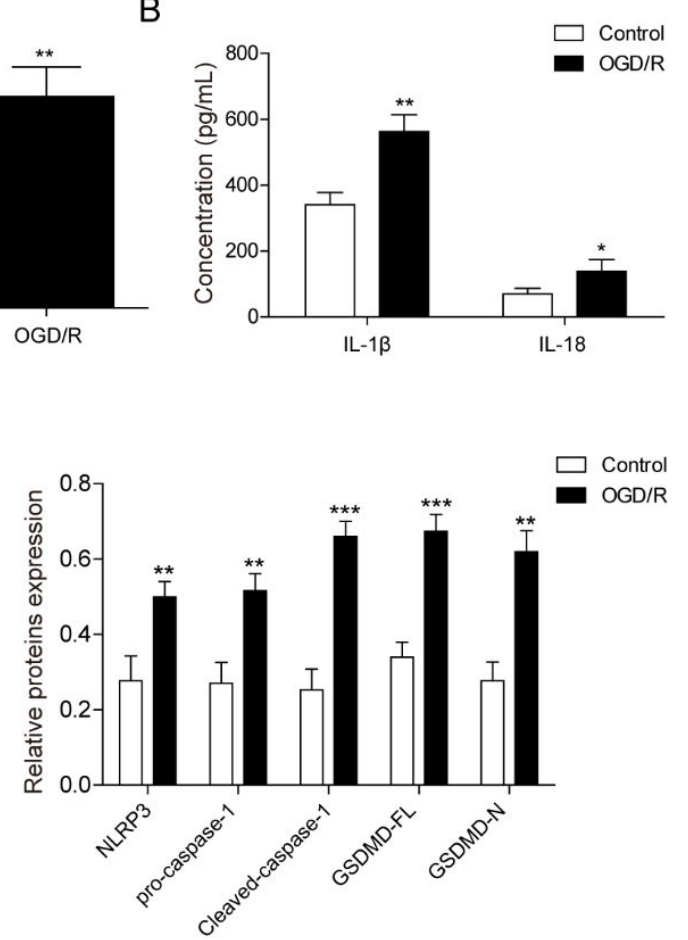

Fig. 1. OGD/R treatment leads to hippocampal neuron pyroptosis. An OGD/R cell model was established in primary hippocampal neurons. (A) Pyroptosis was detected by flow cytometry using caspase- 1 and PI staining. (B) ELISA was applied to measure IL- $1 \beta$ and IL-18 expression. (C) LDH release in the cell supernatant. (D) Pyroptosis-related markers, including NLRP3, pro-caspase-1, cleaved caspase-1, GSDMD-FL, and GSDMD-N, were assessed using western blot assay. The results are shown as the mean \pm SD. $n=3$ per group. ${ }^{*} p<0.05,{ }^{* *} \mathrm{p}<0.01,{ }^{* * *} \mathrm{p}<0.001$.

mate cytotoxicity, and the results revealed an obvious elevation in LDH levels in the OGD/R group (Fig. 1C). In addition, the protein expression of NLRP3, pro-caspase-1, cleaved caspase-1, GSDMDFL and GSDMD-N was upregulated in the OGD/R group (Fig. 1D). These findings suggested that pyroptosis of hippocampal neurons was exacerbated by $\mathrm{OGD} / \mathrm{R}$ treatment.

\section{OA treatment inhibits pyroptosis induced by $O G D / R$}

We next examined the impact of OA on OGD/R-treated neurons. Three concentrations of OA $(0.5,2$, or $8 \mu \mathrm{M})$ were added to the cells, and pyroptosis was assessed using flow cytometry. Our results demonstrated that OGD/R-induced pyroptosis was reduced by OA treatment (Fig. 2A). Moreover, IL-1 $\beta$ and IL-18 levels were noticeably decreased after the same OA treatment as measured by ELISA (Fig. 2B). OGD/R-induced cytotoxicity was restored by $\mathrm{OA}$ application, as reflected by the decreased $\mathrm{LDH}$ release after OA treatment (Fig. 2C). Meanwhile, pyroptosisassociated protein expression was downregulated in hippocampal neurons after OA treatment compared to the OGD/R group (Fig. 2D). These findings demonstrated that OA effectively suppressed hippocampal neuron pyroptosis induced by OGD/R.

\section{$O A$ application reverses the effects of $O G D / R$ on the expression of miR-186-5p and $\mathrm{Ngb}$}

To further validate the in vitro findings and investigate the potential mechanism, miR-186-5p and Ngb expression was measured in neurons exposed to OGD/R with different doses of OA. MiR-186$5 p$ was elevated in the OGD/R group, while its expression declined after treatment with 2 or $8 \mu \mathrm{M} \mathrm{OA}$ (Fig. 3A). The mRNA (Fig. 3B) and protein (Fig. 3C) expression levels of Ngb were decreased in the OGD/R group. In addition, $\mathrm{OA}$ application at 2 or $8 \mu \mathrm{M}$ upregulated the mRNA (Fig. 3B) and protein (Fig. 3C) expression of $\mathrm{Ngb}$. Our data suggested that OA effectively inhibited the increase in miR-186-5p levels and elevated the decrease in Ngb levels in hippocampal neurons induced by OGD/R. Knockdown of miR186-5p inhibits neuronal pyroptosis after OGD/R.

\section{Knockdown of miR-186-5p inhibits neuronal pyroptosis after $O G D / R$}

A miR-186-5p inhibitor was constructed to further explore the role of miR-186-5p in OGD/R-induced neuronal pyroptosis. We discovered that miR-186-5p was silenced by the miR-186-5p inhibitor in neurons (Fig. 4A). Flow cytometry showed that pyroptosis was remarkably inhibited in the OGD/R+miR-186-5p inhibitor 
A
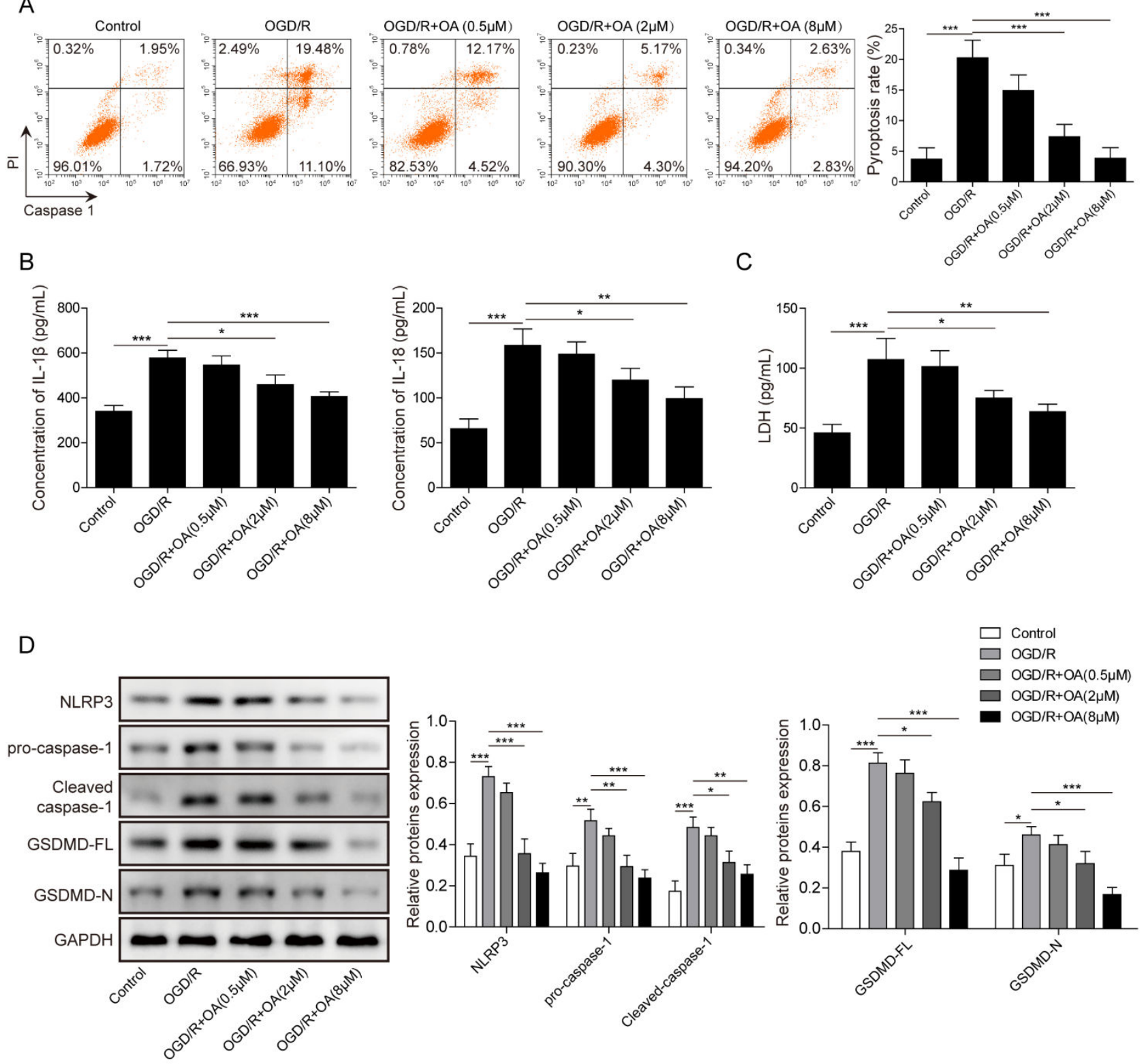

Fig. 2. OGD/R-induced pyroptosis is ameliorated by OA. (A) Flow cytometry was utilized to analyse the impact of OA at different doses on OGD/Rinduced pyroptosis. (B) Levels of IL-1 $\beta$ and IL-18 were analysed by ELISA after OA treatment. (C) LDH release after OA treatment. (D) Expression of pyroptosis-related markers was evaluated using western blot assay. The results are shown as the mean \pm SD. $n=3$ per group. ${ }^{*} \mathrm{p}<0.05,{ }^{* *} \mathrm{p}<0.01,{ }^{* * *} \mathrm{p}<0.001$.

group compared to the OGD/R+inhibitor NC group (Fig. 4B). We also found that knockdown of miR-186-5p led to the downregulation of IL- $1 \beta$ and IL-18 expression (Fig. 4C). In addition, miR-1865 p suppression effectively inhibited OGD/R-induced LDH release (Fig. 4D). Furthermore, western blot data confirmed that upregulation of pyroptosis-related markers, including NLRP3, pro-caspase-1, cleaved caspase-1, GSDMD-FL, and GSDMD-N, induced by OGD/R was evidently decreased after miR-186-5p knockdown (Fig. 4E). Taken together, our results indicated that miR-186-5p knockdown repressed neuronal pyroptosis after OGD/R.

\section{Ngb is a target gene of miR-186-5p}

We next assessed the interaction between miR-186-5p and Ngb. Compared to the mimic NC group, miR-186-5p was increased in primary hippocampal neurons in the miR-186-5p mimic group, suggesting that miR-186-5p mimics were successfully transfected (Fig. 5A). As shown in Fig. 5B and 5C, Ngb mRNA and protein expression was downregulated in the miR-186-5p mimic group and upregulated in the miR-186-5p inhibitor group in neurons. The binding sites of miR-186-5p and Ngb were predicted using webbased bioinformatic software (TargetScan version 7.2) (Fig. 5D). 
A

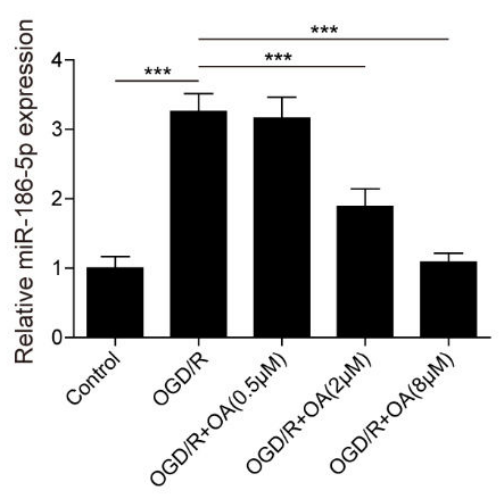

C

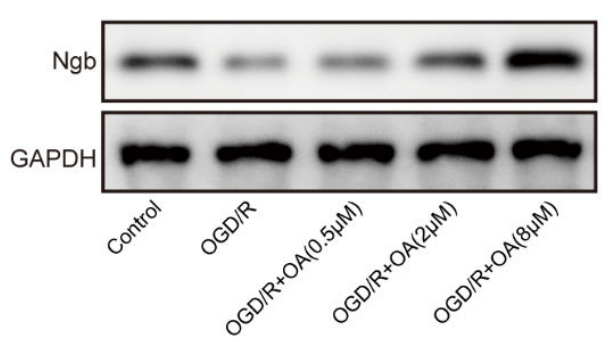

B
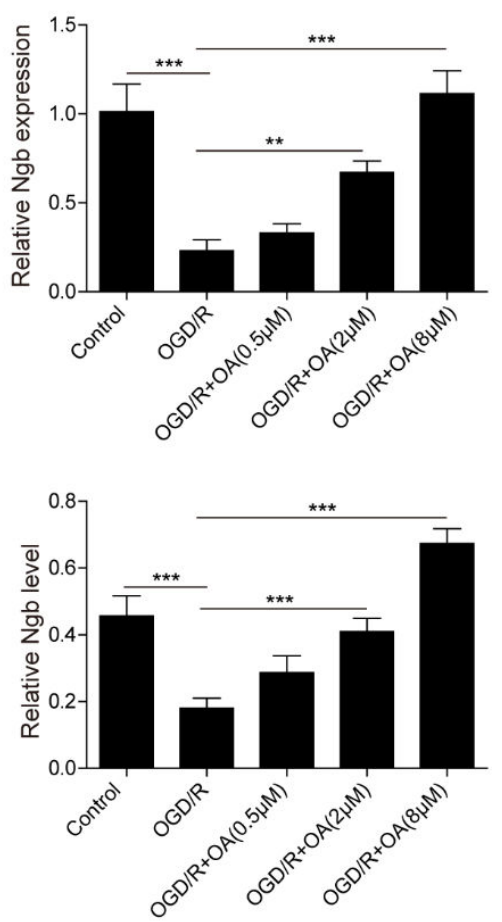

Fig. 3. OA application reverses the levels of miR-186-5p and Ngb caused by OGD/R. (A) The levels of miR-186-5p were evaluated as shown by qRT-PCR. (B, C) The mRNA (B) and protein (C) expression of Ngb was measured using qRT-PCR and western blotting, respectively. The results are shown as the mean \pm SD. $n=3$ per group. ${ }^{* *} \mathrm{p}<0.01,{ }^{* * *} \mathrm{p}<0.001$.

We then further confirmed the direct association between miR186-5p and Ngb. Luciferase activity was downregulated following cotransfection with Ngb-WT and miR-186-5p mimics in hippocampal neurons; however, there was no significant difference when Ngb-MUT and miR-186-5p mimics were cotransfected into cells (Fig. 5D). These findings implied that miR-186-5p bound to $\mathrm{Ngb}$ and inhibited the expression of Ngb.

\section{OA inhibits neuronal pyroptosis by downregulating miR-186-5p and upregulating $\mathrm{Ngb}$}

We further explored the underlying mechanism by which OA suppresses pyroptosis after OGD/R. Expression of Ngb was measured in hippocampal neurons using qRT-PCR and western blot, and the results suggested that Ngb levels were increased after overexpression of $\mathrm{Ngb}$ (Fig. 6A, 6B). The inhibitory effect of OA was examined by flow cytometry. Compared to the OGD/ R group, overexpression of Ngb or inhibition of miR-186-5p enhanced the effect of OA. However, the inhibitory effect of OA on neurons induced by OGD/R was effectively restored after miR186-5p overexpression, and $\mathrm{p}-\mathrm{Ngb}$ reversed this phenomenon (Fig. 6C). Next, we discovered that the downregulation of IL- $1 \beta$ and IL-18 after OA supply was apparently increased in the OGD/
$\mathrm{R}+\mathrm{OA}+\mathrm{miR}-186-5 \mathrm{p}$ mimic group and that Ngb overexpression alleviated this phenotype. However, the expression of IL- $1 \beta$ and IL18 was further inhibited in the $\mathrm{OGD} / \mathrm{R}+\mathrm{OA}+\mathrm{p}-\mathrm{Ngb}$ and $\mathrm{OGD} /$ $\mathrm{R}+\mathrm{OA}+\mathrm{miR}-186-5 \mathrm{p}$ inhibitor groups (Fig. 6D). In line with these results, $\mathrm{LDH}$ release was increased in the $\mathrm{OGD} / \mathrm{R}+\mathrm{OA}+\mathrm{miR}-$ 186-5p mimic group and decreased in the $\mathrm{OGD} / \mathrm{R}+\mathrm{OA}+\mathrm{p}-\mathrm{Ngb}$ and $\mathrm{OGD} / \mathrm{R}+\mathrm{OA}+\mathrm{miR}-186-5 \mathrm{p}$ inhibitor groups compared to the $\mathrm{OGD} / \mathrm{R}+\mathrm{OA}+\mathrm{NC}$ group (Fig. 6E). Finally, we found that miR-186$5 p$ overexpression led to an increase in pyroptosis-related markers, including NLRP3, pro-caspase-1, cleaved caspase-1, GSDMD-FL, and GSDMD-N, compared to OA treatment in OGD/R neurons, and overexpression of Ngb reversed this phenomenon (Fig. 6F). Our results demonstrated that OA inhibited OGD/R-induced neuronal pyroptosis by regulating miR-186-5p by targeting Ngb.

\section{OA suppresses pyroptosis to protect neurons from damage}

To confirm the effects of OA, an MCAO model was constructed in mice. We observed that cerebral ischaemia area was noticeably reduced by $\mathrm{OA}$ administration compared to the MCAO group (Fig. 7A, 7B, Fig. S1). The expression of proinflammatory cytokines, including IL-1 $\beta$ and IL-18 (Fig. 7C), LDH release (Fig. 7D), and the expression of pyroptosis-related markers (Fig. 7E), 


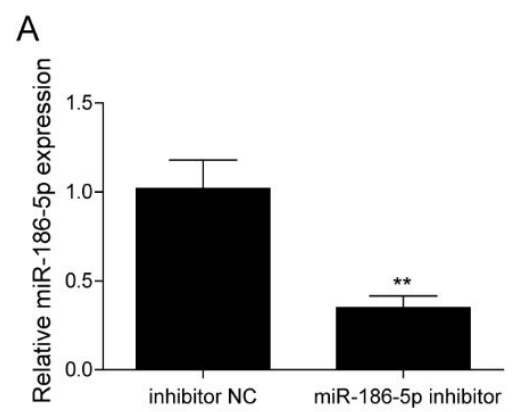

C

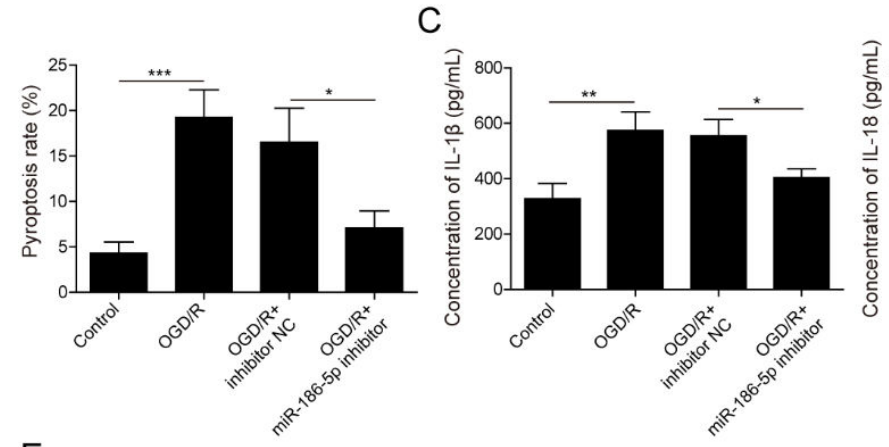

E
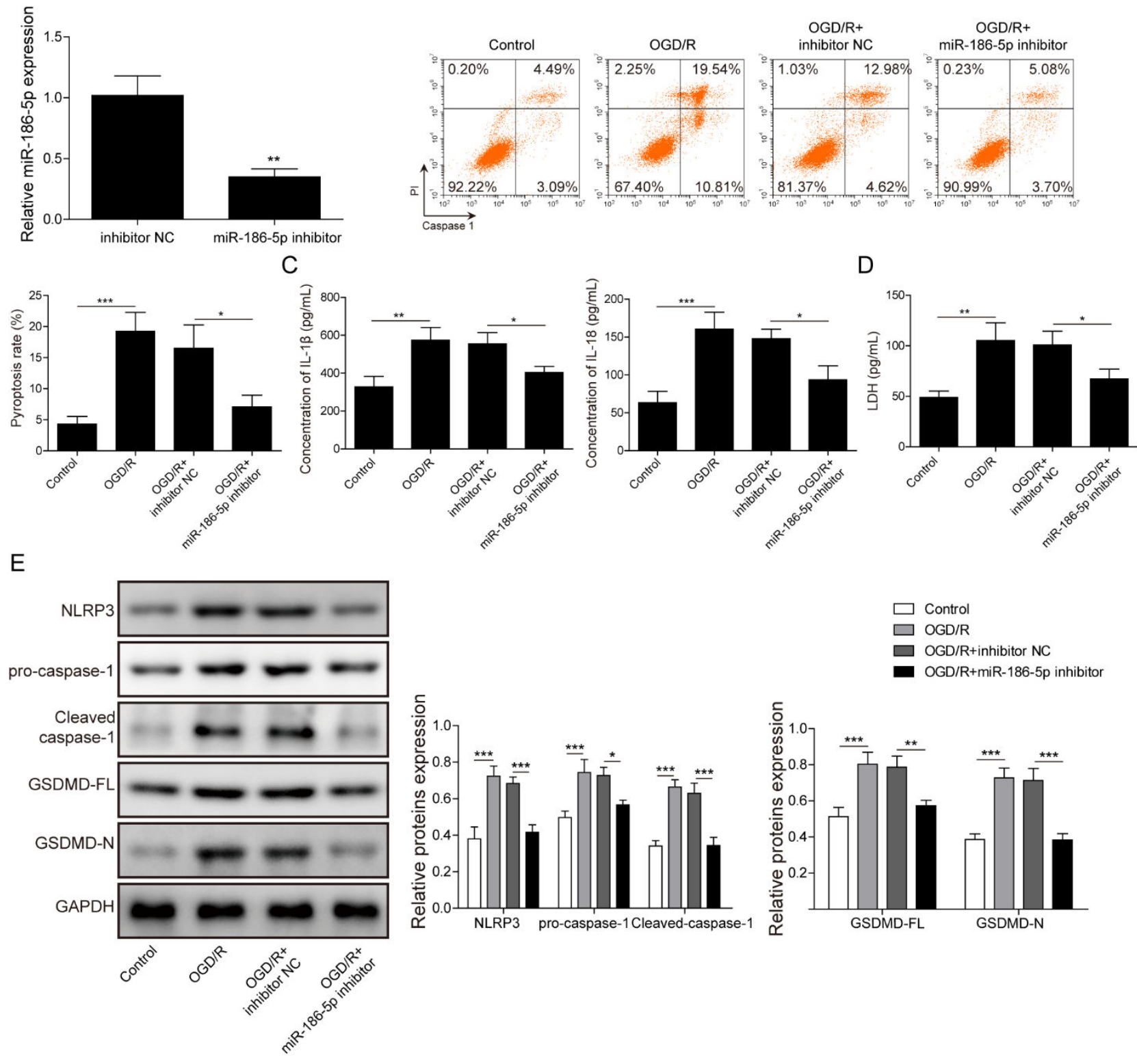

Fig. 4. miR-186-5p knockdown alleviates OGD/R-induced pyroptosis. The miR-186-5p inhibitor or inhibitor NC was transfected into neurons. (A) Expression of miR-186-5p was measured using qRT-PCR. (B) Pyroptosis was assessed using flow cytometry. (C) Expression of IL-1 13 and IL-18 was detected by ELISA. (D) LDH release was restored by inhibition of miR-186-5p. (E) Pyroptosis-related markers were detected by western blot assay. The results are shown as the mean $\pm S D$. $n=3$ per group. ${ }^{*} p<0.05,{ }^{* *} p<0.01,{ }^{* * *} p<0.001$.

in the $\mathrm{MCAO}+\mathrm{OA}$ group were significantly inhibited compared with those in the MCAO group. Furthermore, we observed that the increase in miR-186-5p levels in MCAO mice was remarkably inhibited in response to OA treatment (Fig. 7F). Meanwhile, Ngb expression in MCAO mice was decreased but was noticeably upregulated after OA treatment at the mRNA and protein levels (Fig. 7G, 7H). Altogether, we demonstrated that OA inhibited MCAOinduced neuronal pyroptosis to protect the brain from ischaemia reperfusion injury.

\section{DISCUSSION}

Stroke, the primary cause of long-term disability, has become one of the top reasons for mortality worldwide [4]. Many mechanisms participate in stroke-induced disorders, such as mitochondrial death pathways, excitotoxicity, inflammation, apoptosis, necrosis and free radical release [30]. Currently, emerging evidence has revealed that pyroptosis, a caspase-1-dependent cell death process that produces pro forms of cytokines and accelerates their release, 


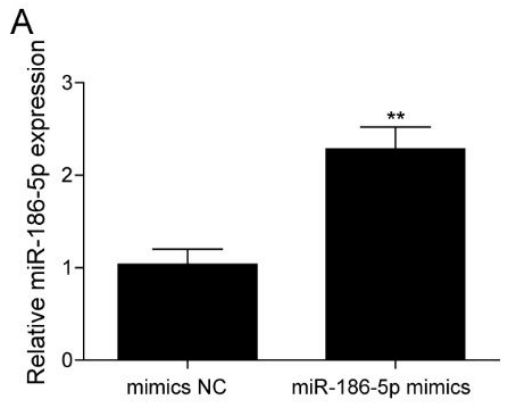

C

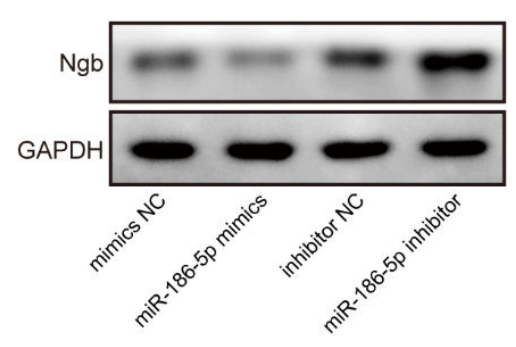

D
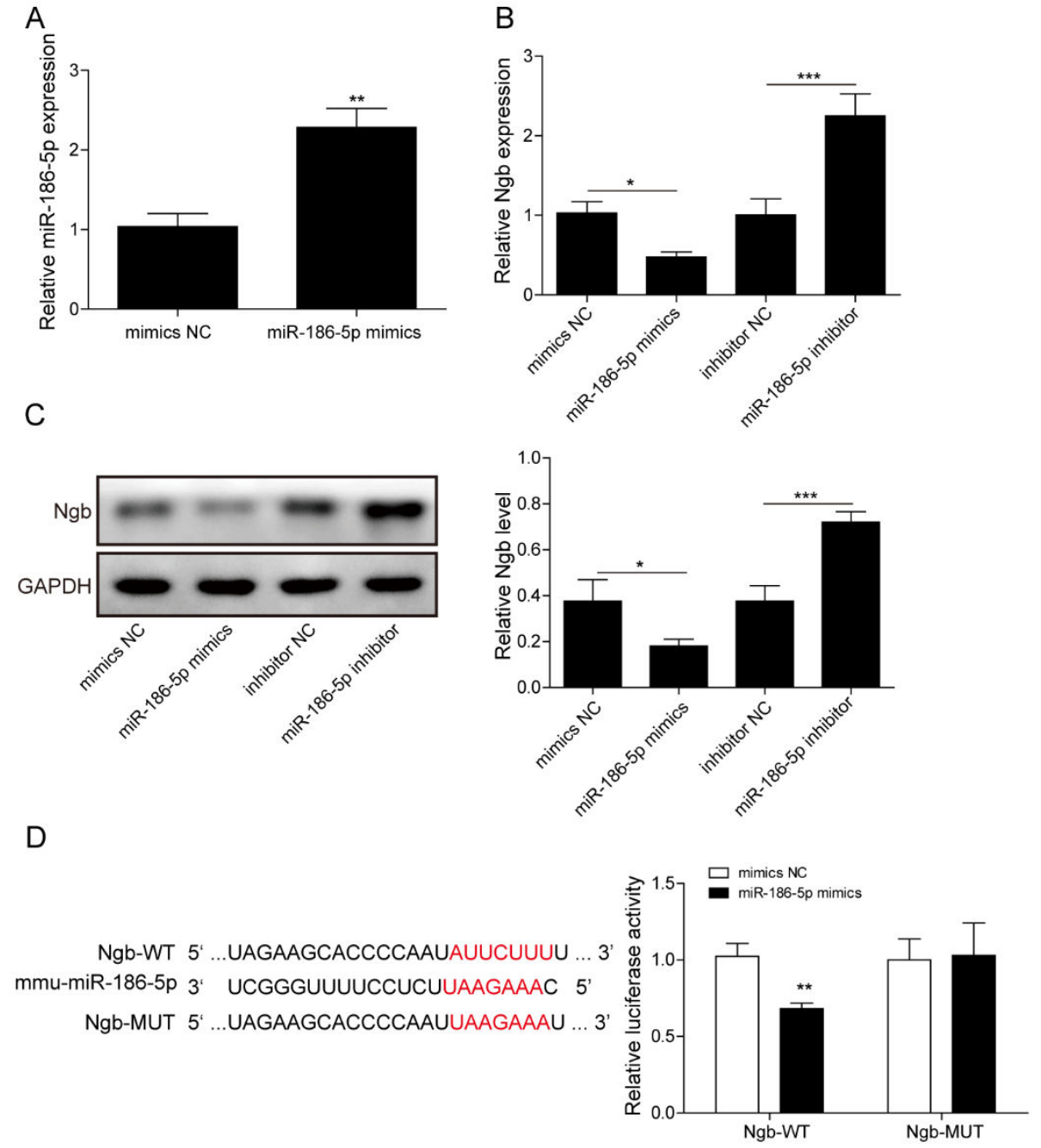

Fig. 5. miR-186-5p overexpression inhibits Ngb expression. (A) qRT-PCR was used to evaluate the level of miR-186-5p after neurons were incubated with miR-186-5p mimics. (B, C) mRNA (B) and protein (C) levels of Ngb were detected by qRT-PCR and western blotting in neurons. (D) The binding site was predicted using TargetScan version 7.2, and luciferase activity was measured using double luciferase reporter assay in hippocampal neurons. The results are shown as the mean \pm SD. $n=3$ per group. ${ }^{*} \mathrm{p}<0.05,{ }^{* *} \mathrm{p}<0.01,{ }^{* * *} \mathrm{p}<0.001$.

may be one of the primary neuron death mechanisms in stroke [31]. However, further investigation of the molecular mechanisms of pyroptosis is still required, and therapeutic strategies targeting pyroptosis would present novel opportunities for stroke. The present study provides strong evidence that OA exerts a crucial effect in alleviating neuronal pyroptosis and cerebral infarction following stroke. Furthermore, we observed that OGD/R-induced upregulation of miR-186-5p and downregulation of $\mathrm{Ngb}$ could be reversed by OA. To the best of our knowledge, we demonstrated for the first time that OA reduced miR-186-5p to inhibit pyroptosis via upregulation of $\mathrm{Ngb}$, with a corresponding reduction in proinflammatory cytokines and LDH release.

$\mathrm{OA}$ is a pentacyclic triterpenoid extracted and isolated from plants [32]. Recently, emerging discoveries have provided insights into the probable therapeutic impacts of $\mathrm{OA}$ on different diseases and their symptoms. Regarding neurodegenerative disorders, the protective activities of $\mathrm{OA}$ have been used to test improvement of motor performance and to slow disease progression in animal models of amyotrophic lateral sclerosis and multiple sclerosis [33, 34]. It has been verified that $\mathrm{OA}$ extracted from $\mathrm{A}$. cordata reduces neuronal death induced by $\mathrm{H}_{2} \mathrm{O}_{2}$ treatment and increases reactive oxygen species (ROS) generation and intracellular calcium concentration, indicating that A. cordata exerting its neuroprotective role might be partially attributable to this compound [35]. More importantly, a recent study illustrated that decreases in neuronal degeneration and glial reaction are observed when $\mathrm{OA}$ is pre-treated in adult rats with focal cortical hypoxia after brain injection of cobalt chloride [36]. It has also been reported that OA 

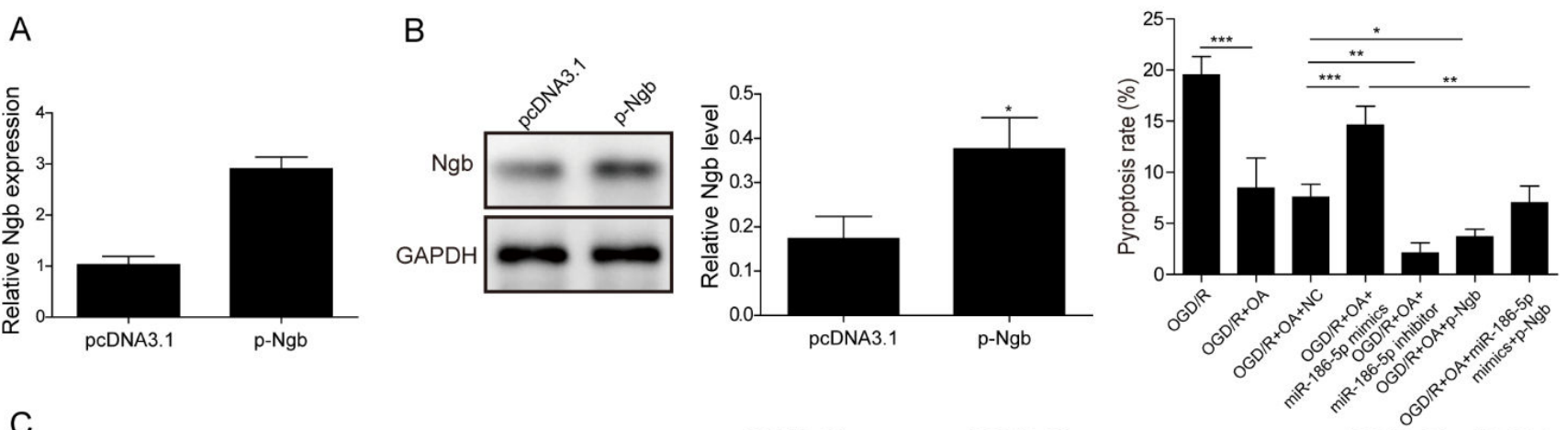

C
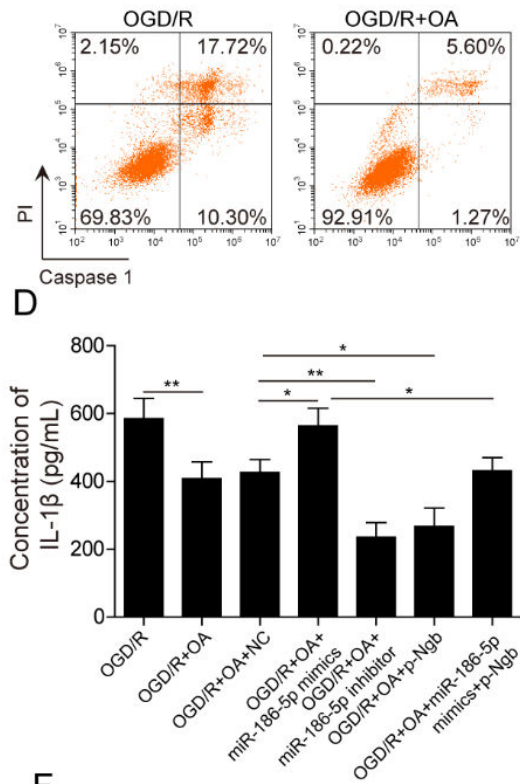

F
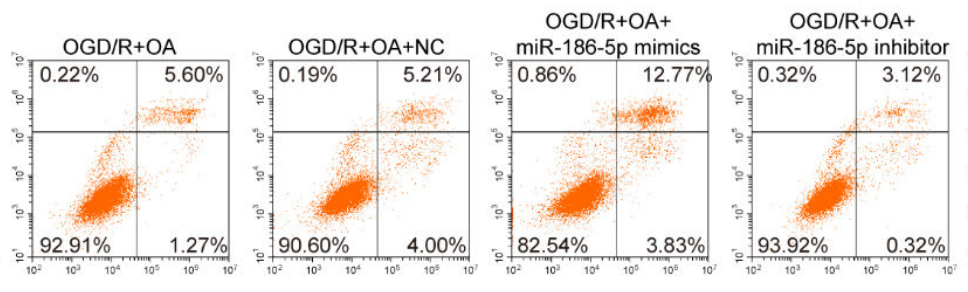

\section{E}

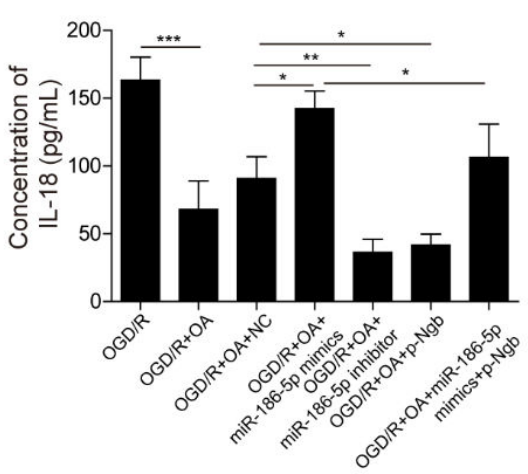

OGD/R+OA+miR-186-5p

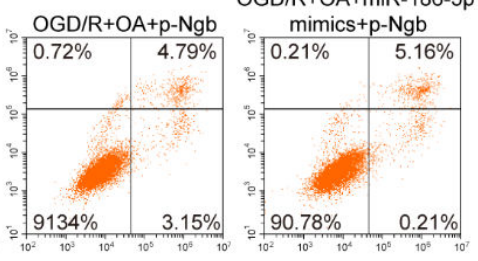

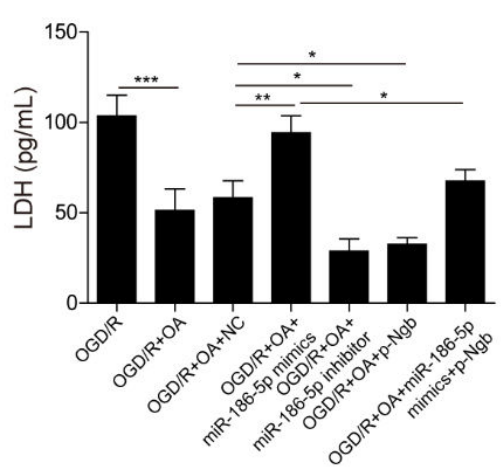
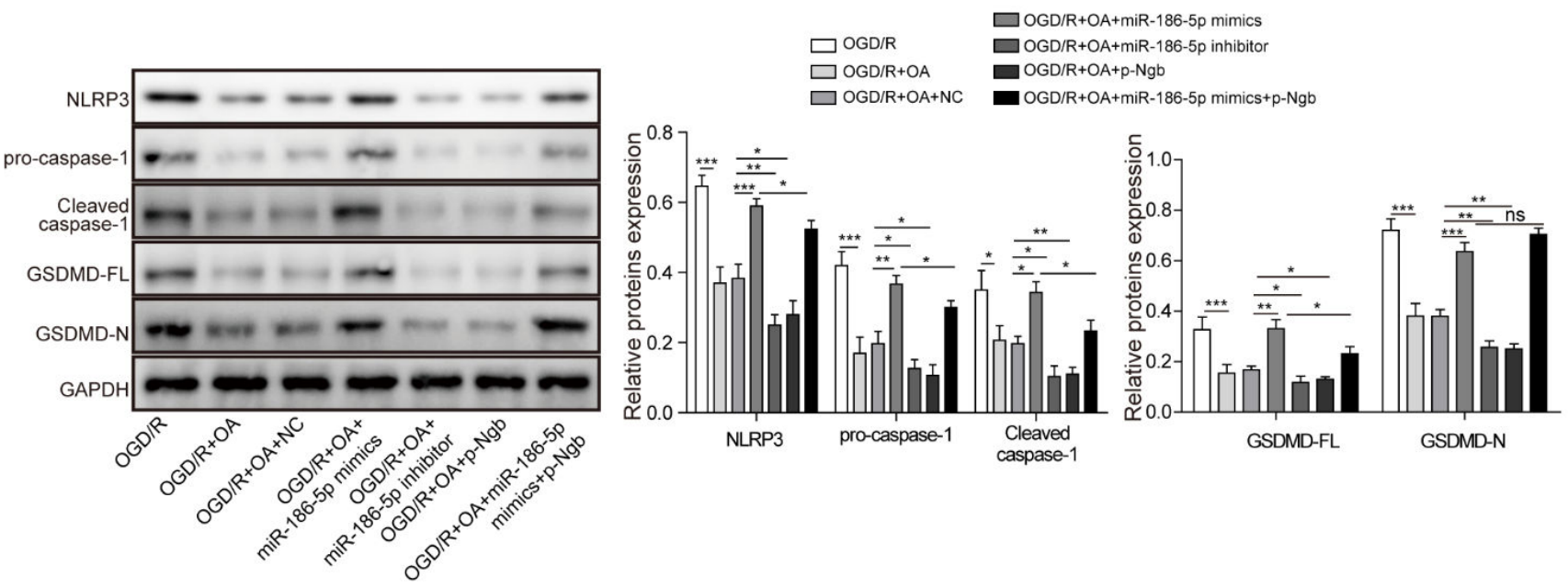

Fig. 6. OA suppresses pyroptosis induced by OGD/R by regulating miR-186-5p and Ngb expression. (A, B) mRNA (A) and protein (B) expression levels of $\mathrm{Ngb}$ in neurons were evaluated as shown by qRT-PCR and western blotting. (C) Pyroptosis was detected by flow cytometry. (D) Alterations in IL$1 \beta$ and IL-18 were determined by ELISA. (E) LDH release was modulated after OA treatment in OGD/R neurons. (F) Pyroptosis-related markers were examined by western blotting. The results are shown as the mean \pm SD. $n=3$ per group. ${ }^{*} \mathrm{p}<0.05,{ }^{* *} \mathrm{p}<0.01,{ }^{* * *} \mathrm{p}<0.001$. 
A

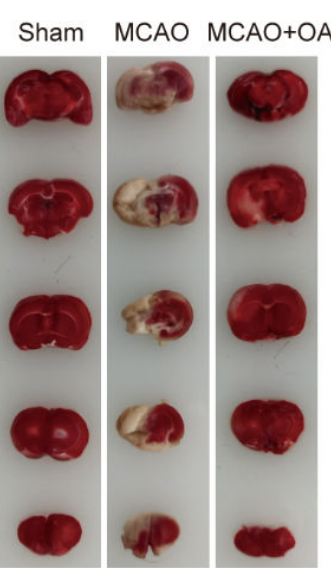

D

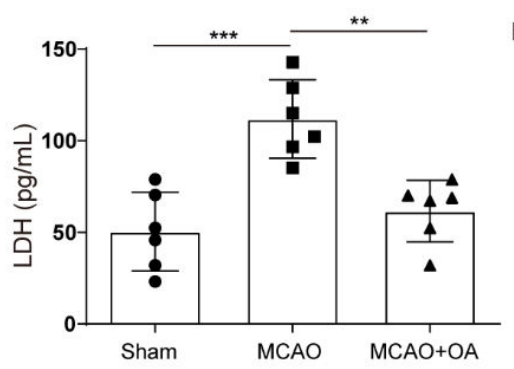

B

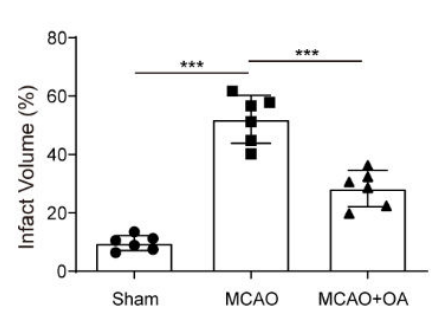

E

C
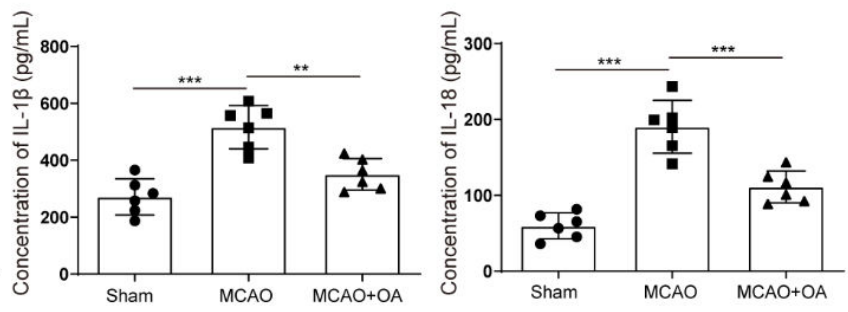

- Sham

- MCAO

- MCAO+OA

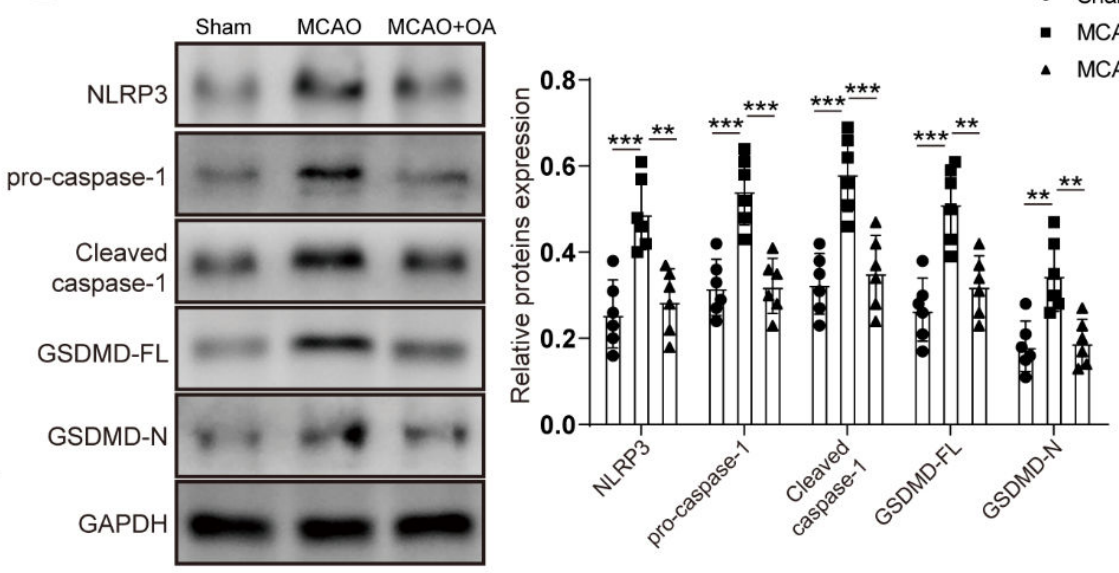

$\mathrm{F}$

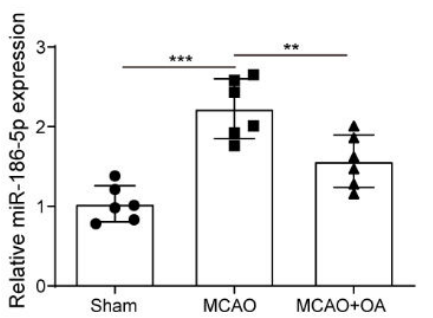

G

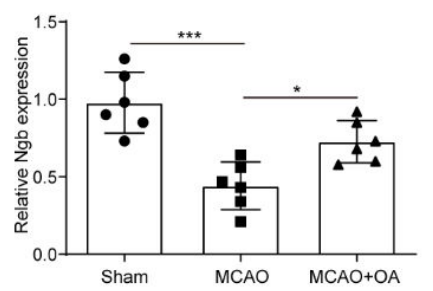

$\mathrm{H}$
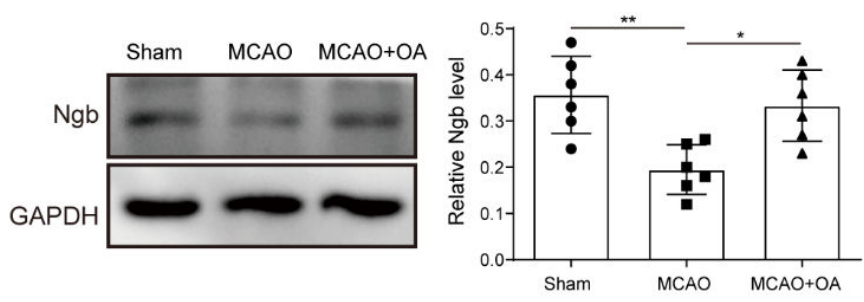

Fig. 7. The effect of $\mathrm{OA}$ treatment in $\mathrm{MCAO} / \mathrm{R}$ mice. $\mathrm{MCAO} / \mathrm{R}$ or sham surgery was performed in mice, and $\mathrm{OA}$ was administered to the $\mathrm{MCAO} /$ R+OA group. (A) TTC was utilized to detect the cerebral ischaemia area. (B) Quantification of infarct volume. (C) IL- $1 \beta$ and IL-18 levels were determined using ELISA. (D) LDH release. (E) Pyroptosis-related markers were evaluated by western blot assay. (F) miR-186-5p expression was examined by qRT-PCR. $(\mathrm{G}, \mathrm{H}) \mathrm{mRNA}(\mathrm{G})$ and protein $(\mathrm{H})$ levels of Ngb were evaluated by qRT-PCR and western blotting, respectively. The results are shown as the mean \pm SD. $n=6$ per group. ${ }^{*} \mathrm{p}<0.05,{ }^{* *} \mathrm{p}<0.01,{ }^{* * *} \mathrm{p}<0.001$.

preadministration prolongs survival time, alleviates cerebral oedema, infarct area and neurological function in mice with bilateral common carotid artery ligation (BCCAL), obviously promotes cell survival, improves SOD activity, and reduces LDH and MDA levels in $\mathrm{H}_{2} \mathrm{O}_{2}$-injured PC12 cells [11]. Pyroptosis is one of the principal cell death mechanisms in neurons during stroke [37]. In the present report, we provided compelling evidence that pyroptosis was inhibited by OA in primary hippocampal neurons during $\mathrm{OGD} / \mathrm{R}$. In addition, a previous study indicated that after $\mathrm{MCAO} /$ $\mathrm{R}$, the NLRP3 inflammasome is assembled and activated, and the $\mathrm{N}$-terminal fragment of gasdermin D (GSDMD) is cleaved to induce pyroptosis [31]. We explored the molecular evidence of pyroptosis observed in neurons, and we identified upregulation of NLRP3, accumulation of caspase-1 and GSDMD, release of inflammasome-dependent cytokines (IL-1 $\beta$ and IL-18), and extravasation of $\mathrm{LDH}$, all of which were prevented by treatment with $\mathrm{OA}$ in a dose-dependent manner. Although experimental data are still required, our existing data demonstrate the beneficial consequences of $\mathrm{OA}$ for the prevention or treatment of ischaemic stroke. Certainly, the precise mechanism is still deficient and needs further investigation.

Currently, growing evidence has proven that miRNAs play criti- 
cal roles during ischaemic stroke. In the current study, we observed elevated miR-186-5p levels in hippocampal neurons exposed to $\mathrm{OGD} / \mathrm{R}$. This is consistent with a previous report illustrating that miR-186-5p was noticeably increased in SH-SY5Y cells suffering from OGD/R [4]. Early in vitro data suggest that increased miR186-5p levels can induce apoptosis [25], prompting us to explore whether miR-186-5p regulates pyroptosis. To the best of our knowledge, this is the first time that miR-186-5p knockdown has been shown to inhibit neuronal pyroptosis in an ischaemic stroke cell model. Previous studies have demonstrated that $\mathrm{OA}$ can attenuate disease through the regulation of miRNAs. For example, antagonizing the function of miR-503 with antagomir-503-5p attenuates the inhibitory impact of $\mathrm{OA}$ on osteoclastogenesis [38]. In addition, the hypolipidaemic function of $\mathrm{OA}$ is regulated by the miR-98-5p/peroxisome proliferator-activated receptor- $\gamma$ coactivator-1 $\beta$ (PGC-1 $\beta$ ) pathway in hyperlipidaemic mice induced by a high-fat diet [23]. In chronic unpredictable mild stress (CUMS), the role of miR-132 in the modulation of OA functions to ameliorate anhedonic and anxiogenic behaviours in male mice is mediated via BDNF-ERK-CREB signalling [22]. At the same time, we discovered that $\mathrm{OA}$ reduced cerebral ischaemia and diminished pyroptosis by inhibiting the upregulation of miR-186-5p induced by OGD/R. Taken together, our present observations discovered pyroptosis suppression caused by OA treatment through miRNA regulation during ischaemic stroke, illustrating the potential therapeutic function of $\mathrm{OA}$.

Recently, accumulating evidence has demonstrated that Ngb, a tissue globin expressed specifically in brain neurons, plays a protective role against stroke and related neurological disorders. High expression of the endogenous protein Ngb is linked with preserved mitochondrial function and elevated nerve cell survival both in vitro and in vivo [39]. In Ngb-overexpressing transgenic (Ngb-Tg) mice, brain infarction volumes after transient focal cerebral ischaemia are apparently decreased [40]. Moreover, Ngb overexpression reduces OGD-induced neurotoxicity by binding to the mitochondrial complex III subunit Cyc1 [41]. During hypoxia, the redox state of Ngb regulates the stabilization of hypoxiainducible factor-1 (HIF-1) and nuclear factor-erythroid 2-related factor 2 (Nrf2) and the release of cytochrome $\mathrm{C}$ in neuronal cells [42]. In addition, Ngb is involved in the neuroprotection of hypoxic postconditioning (HPC) against transient global cerebral ischaemia (tGCI) via maintenance of $\mathrm{Na}^{+} / \mathrm{K}^{+}$ATPase activity in the hippocampal CA1 [15]. Our data suggested that the decreased $\mathrm{Ngb}$ induced by OGD/R was elevated after OA application at the mRNA and protein levels, illustrating its critical role in OA treatment. Subsequently, a luciferase assay further confirmed the direct interaction between miR-186-5p and Ngb. A novel artificial recombinant protein, Mn-TAT PTD-Ngb, upregulates Nrf2, which improves pyroptosis and protects against oxidative stress [21]. Our work first illustrated that the impact of $\mathrm{OA}$ in alleviating $\mathrm{OGD} / \mathrm{R}$ induced pyroptosis could be reversed by miR-186-5p overexpression or Ngb knockdown. Taken together, we demonstrated that OA downregulates miR-186-5p to restrain pyroptosis by affecting $\mathrm{Ngb}$ expression, resulting in a protective role against ischaemic stroke.

In conclusion, we identified $\mathrm{OA}$ as an effective factor for improving cerebral ischaemia/reperfusion injury. Briefly, OA reduces miR-186-5p expression, upregulates the targeted Ngb protein levels, inhibits neuronal pyroptosis, and consequently plays a neuroprotective role in cerebral ischaemia. Collectively, our findings offer unique perspectives on the curative mechanism of $\mathrm{OA}$ and treatment options for ischaemic stroke.

\section{ACKNOWLEDGEMENTS}

We would like to give our sincere gratitude to the reviewers for their constructive comments. This work was supported by a grant from the Huaihua Science and Technology Planning Project (No.2020R3105), a grant from the Scientific Research Fund of Hunan Provincial Education Department (Grant No.20A356), and a grant from the National Natural Science Foundation of China (Grant No.81703821).

\section{CONFLICT OF INTEREST}

The authors declare that there is no conflict of interest.

\section{AUTHORS' CONTRIBUTION}

Conception and study design: SCC. Data acquisition: XPL. Data analysis: XL, GYT. Manuscript drafting: LMY. Manuscript revising: $\mathrm{XSH}$. All authors have read and approved the final version of this manuscript to be published.

\section{REFERENCES}

1. Hankey GJ (2017) Stroke. Lancet 389:641-654.

2. Meschia JF, Brott T (2018) Ischaemic stroke. Eur J Neurol 25:35-40.

3. Yang Q, Huang Q, Hu Z, Tang X (2019) Potential neuroprotective treatment of stroke: targeting excitotoxicity, oxidative stress, and inflammation. Front Neurosci 13:1036.

4. Phipps MS, Cronin CA (2020) Management of acute ischemic stroke. BMJ 368:16983. 
5. Muresanu DF, Strilciuc S, Stan A (2019) Current drug treatment of acute ischemic stroke: challenges and opportunities. CNS Drugs 33:841-847.

6. Zhang W, Feng J, Cheng B, Lu Q, Chen X (2018) Oleanolic acid protects against oxidative stress-induced human umbilical vein endothelial cell injury by activating AKT/eNOS signaling. Mol Med Rep 18:3641-3648.

7. Kashyap D, Sharma A, Tuli HS, Punia S, Sharma AK (2016) Ursolic acid and oleanolic acid: pentacyclic terpenoids with promising anti-inflammatory activities. Recent Pat Inflamm Allergy Drug Discov 10:21-33.

8. Zhang L, Xia R, Jia J, Wang L, Li K, Li Y, Zhang J (2018) Oleanolic acid protects against cognitive decline and neuroinflammation-mediated neurotoxicity by blocking secretory phospholipase A2 IIA-activated calcium signals. Mol Immunol 99:95-103.

9. Wang JL, Ren CH, Feng J, Ou CH, Liu L (2020) Oleanolic acid inhibits mouse spinal cord injury through suppressing inflammation and apoptosis via the blockage of p38 and JNK MAPKs. Biomed Pharmacother 123:109752.

10. An Q, Hu Q, Wang B, Cui W, Wu F, Ding Y (2017) Oleanolic acid alleviates diabetic rat carotid artery injury through the inhibition of NLRP3 inflammasome signaling pathways. Mol Med Rep 16:8413-8419.

11. Rong ZT, Gong XJ, Sun HB, Li YM, Ji H (2011) Protective effects of oleanolic acid on cerebral ischemic damage in vivo and $\mathrm{H}(2) \mathrm{O}(2)$-induced injury in vitro. Pharm Biol 49:78-85.

12. Yu Z, Liu N, Liu J, Yang K, Wang X (2012) Neuroglobin, a novel target for endogenous neuroprotection against stroke and neurodegenerative disorders. Int J Mol Sci 13:6995-7014.

13. Xiong XX, Pan F, Chen RQ, Hu DX, Qiu XY, Li CY, Xie XQ, Tian B, Chen XQ (2018) Neuroglobin boosts axon regeneration during ischemic reperfusion via p38 binding and activation depending on oxygen signal. Cell Death Dis 9:163.

14. Yu Z, Cheng C, Liu Y, Liu N, Lo EH, Wang X (2018) Neuroglobin promotes neurogenesis through Wnt signaling pathway. Cell Death Dis 9:945.

15. Wen H, Liu L, Zhan L, Liang D, Li L, Liu D, Sun W, Xu E (2018) Neuroglobin mediates neuroprotection of hypoxic postconditioning against transient global cerebral ischemia in rats through preserving the activity of $\mathrm{Na}^{+} / \mathrm{K}^{+}$ATPases. Cell Death Dis 9:635.

16. Ye A, Li W, Zhou L, Ao L, Fang W, Li Y (2020) Targeting pyroptosis to regulate ischemic stroke injury: molecular mechanisms and preclinical evidences. Brain Res Bull 165:146-160.

17. Liang Y, Song P, Chen W, Xie X, Luo R, Su J, Zhu Y, Xu J, Liu R, Zhu P, Zhang Y, Huang M (2021) Inhibition of caspase-1 ameliorates ischemia-associated blood-brain barrier dysfunction and integrity by suppressing pyroptosis activation. Front Cell Neurosci 14:540669.

18. Sun R, Peng M, Xu P, Huang F, Xie Y, Li J, Hong Y, Guo H, Liu Q, Zhu W (2020) Low-density lipoprotein receptor (LDLR) regulates NLRP3-mediated neuronal pyroptosis following cerebral ischemia/reperfusion injury. J Neuroinflammation $17: 330$

19. Wang M, Liu Z, Hu S, Duan X, Zhang Y, Peng C, Peng D, Han L (2020) Taohong Siwu decoction ameliorates ischemic stroke injury via suppressing pyroptosis. Front Pharmacol 11:590453.

20. Wang Y, Zhang P, Yuan M, Li X (2019) Overexpression of miRNA-21 promotes the proliferation and invasion in hepatocellular carcinoma cells via suppressing SMAD7. Technol Cancer Res Treat 18:1533033819878686.

21. Zhang C, Yang R, Hao X, Geng Z, Wang Z (2020) Mn-TAT PTD-Ngb ameliorates inflammation through the elimination of damaged mitochondria and the activation of Nrf2-antioxidant signaling pathway. Biochem Pharmacol 178:114055.

22. Yi LT, Li J, Liu BB, Luo L, Liu Q, Geng D (2014) BDNF-ERKCREB signalling mediates the role of miR-132 in the regulation of the effects of oleanolic acid in male mice. J Psychiatry Neurosci 39:348-359.

23. Chen S, Wen X, Zhang W, Wang C, Liu J, Liu C (2017) Hypolipidemic effect of oleanolic acid is mediated by the miR98-5p/PGC-1 $\beta$ axis in high-fat diet-induced hyperlipidemic mice. FASEB J 31:1085-1096.

24. Mirzaei H, Momeni F, Saadatpour L, Sahebkar A, Goodarzi M, Masoudifar A, Kouhpayeh S, Salehi H, Mirzaei HR, Jaafari MR (2018) MicroRNA: relevance to stroke diagnosis, prognosis, and therapy. J Cell Physiol 233:856-865.

25. Wang R, Bao H, Zhang S, Li R, Chen L, Zhu Y (2018) miR186-5p Promotes apoptosis by targeting IGF-1 in SH-SY5Y OGD/R model. Int J Biol Sci 14:1791-1799.

26. Yu Y, Wu X, Pu J, Luo P, Ma W, Wang J, Wei J, Wang Y, Fei Z (2018) Lycium barbarum polysaccharide protects against oxygen glucose deprivation/reoxygenation-induced apoptosis and autophagic cell death via the PI3K/Akt/mTOR signaling pathway in primary cultured hippocampal neurons. Biochem Biophys Res Commun 495:1187-1194.

27. Chiang T, Messing RO, Chou WH (2011) Mouse model of middle cerebral artery occlusion. JVis Exp 48:2761.

28. Shi YJ, Sun LL, Ji X, Shi R, Xu F, Gu JH (2021) Neuroprotective effects of oleanolic acid against cerebral ischemia-reperfusion injury in mice. Exp Neurol 343:113785.

29. Raskova J, Czerwinski DK, Shea SM, Raska K Jr (1986) Cel- 
lular immunity and lymphocyte populations in developing uremia in the rat. J Exp Pathol 2:229-245.

30. Sekerdag E, Solaroglu I, Gursoy-Ozdemir Y (2018) Cell death mechanisms in stroke and novel molecular and cellular treatment options. Curr Neuropharmacol 16:1396-1415.

31. Barrington J, Lemarchand E, Allan SM (2017) A brain in flame; do inflammasomes and pyroptosis influence stroke pathology? Brain Pathol 27:205-212.

32. Ayeleso TB, Matumba MG, Mukwevho E (2017) Oleanolic acid and its derivatives: biological activities and therapeutic potential in chronic diseases. Molecules 22:1915.

33. Martín R, Carvalho-Tavares J, Hernández M, Arnés M, RuizGutiérrez V, Nieto ML (2010) Beneficial actions of oleanolic acid in an experimental model of multiple sclerosis: a potential therapeutic role. Biochem Pharmacol 79:198-208.

34. Martín R, Hernández M, Córdova C, Nieto ML (2012) Natural triterpenes modulate immune-inflammatory markers of experimental autoimmune encephalomyelitis: therapeutic implications for multiple sclerosis. Br J Pharmacol 166:17081723.

35. Cho SO, Ban JY, Kim JY, Ju HS, Lee IS, Song KS, Bae K, Seong YH (2009) Anti-ischemic activities of aralia cordata and its active component, oleanolic acid. Arch Pharm Res 32:923932.

36. Caltana L, Rutolo D, Nieto ML, Brusco A (2014) Further evidence for the neuroprotective role of oleanolic acid in a model of focal brain hypoxia in rats. Neurochem Int 79:7987.

37. Zhang D, Qian J, Zhang P, Li H, Shen H, Li X, Chen G (2019) Gasdermin D serves as a key executioner of pyroptosis in experimental cerebral ischemia and reperfusion model both in vivo and in vitro. J Neurosci Res 97:645-660.

38. Xie BP, Shi LY, Li JP, Zeng Y, Liu W, Tang SY, Jia LJ, Zhang J, Gan GX (2019) Oleanolic acid inhibits RANKL-induced osteoclastogenesis via ER alpha/miR-503/RANK signaling pathway in RAW264.7 cells. Biomed Pharmacother 117:109045.

39. Guidolin D, Tortorella C, Marcoli M, Maura G, Agnati LF (2016) Neuroglobin, a factor playing for nerve cell survival. Int J Mol Sci 17:1817.

40. Wang X, Liu J, Zhu H, Tejima E, Tsuji K, Murata Y, Atochin DN, Huang PL, Zhang C, Lo EH (2008) Effects of neuroglobin overexpression on acute brain injury and long-term outcomes after focal cerebral ischemia. Stroke 39:1869-1874.

41. Yu Z, Zhang Y, Liu N, Yuan J, Lin L, Zhuge Q, Xiao J, Wang $X$ (2016) Roles of neuroglobin binding to mitochondrial complex III subunit cytochrome $\mathrm{cl}$ in oxygen-glucose deprivation-induced neurotoxicity in primary neurons. Mol Neurobiol 53:3249-3257.

42. Hota KB, Hota SK, Srivastava RB, Singh SB (2012) Neuroglobin regulates hypoxic response of neuronal cells through Hif-1 $\alpha$ - and Nrf2-mediated mechanism. J Cereb Blood Flow Metab 32:1046-1060. 